\section{Analysis of serum glutamic pyruvic transaminase and serum glutamic oxaloacetic transaminase levels in tuberculosis patients who are undergoing oat treatment in Kendari City General Hospital, Kota Kendari, Indonesia}

\author{
Sri Anggarini Rasyid, ${ }^{1}$ Armayani, ${ }^{2}$ \\ Yuniati, ${ }^{1}$ Tiara Mayang Pratiwi Lio ${ }^{1}$ \\ ${ }^{1}$ D-IV Medical Laboratory Technology; \\ ${ }^{2}$ STIKES Mandala Waluya Kendari, \\ Kendari, Indonesia
}

\begin{abstract}
In the treatment of tuberculosis (TB), the patient is generally directly given Anti Tuberculosis Drugs (Obat Anti Tuberculosis/OAT) without examining Serum Glutamic Pyruvic Transaminase (SGPT) and Serum Glutamic Oxaloacetic Transaminase (SGOT) to see whether or not there is liver damage before treatment. Because of the side effects by OAT, it is important to know how the condition of the liver function of TB patients who consume OAT in Kendari City General Hospital (Rumah Sakit Umum Daerah/RSUD) Kota Kendari by looking at SGOT and SGPT levels in order to provide maximum treatment to TB patients. The method of this research was descriptive analysis with cross sectional approach. The sample is all patients had previously been diagnosed with TB by a doctor based on medical records by in RSUD Kota Kendari after 1-2 months OAT treatment without any sign of liver injury before. Examination of SGOT and SGPT levels was performed on all samples in the Laboratory of RSUD Kota Kendari using clinical chemistry analyzer. The results of this study showed that $20 \%$ patients with TB had elevated levels of SGPT and SGOT with average SGOT is $51 \mathrm{U} / \mathrm{L}$ and SGPT is 42.5. The Inference of this study that there is an increase in SGOT and SGPT levels in patients who consume OAT 1-2 months in RSUD Kota Kendari. Elevated levels of SGPT and SGOT due to Anti Tuberculosis (OAT) drugs did not occur in all patients and only increased in 3 people, but qualitatively the effect of administering anti-tuberculosis drugs significantly increased levels of SGPT and SGPT. Thus, monitoring of liver physiology remains recommended especially for elderly patients.
\end{abstract}

\section{Introduction}

Tuberculosis (TB) is an infectious disease caused by Mycobacterium tuberculosis. This bacterium is meant in gram-positive and rod-shaped groups that are resistant to acids. Mycobacterium tuberculosis attacks the lungs and a small number of other organs in the body. Mycobacterium tuberculosis enters the body through the respiratory tract, digestive tract, and open wounds on the skin. Most TB infections occur through the air, namely through inhalation of droplets containing tubercle bacilli originating from an infected person. ${ }^{1}$

$\mathrm{TB}$ is an infectious disease that is still a global concern, although TB control efforts have been implemented in many countries since 1995. Indonesia is one of the countries with a high prevalence of TB cases in the world. It has the third largest number of TB cases in the world. In Indonesia there is one new tuberculosis patient exist every minute, there are 2 smear positive new cases every two minutes and there is one death due to TB every 4 minutes. ${ }^{2}$ Based on the results of the TB prevalence survey in 2013-2014 the TB prevalence rate in Indonesia is $660 / 100,000$ population. This makes Indonesia ranked second in the world with a high TB burden in the world. ${ }^{3}$

Cases of positive pulmonary $\mathrm{TB}$ in Kendari City have increased every year. In 2016, there were 447 pulmonary TB participants taking Anti Tuberculosis Drugs (Obat Anti Tuberculosis/OAT). 2017, patients with pulmonary TB who consumed Category I OAT increased to 467 people and in 2018 increased again to 559 people. The City Hospital of Kendari City also experienced an increase in cases for case finding in the working area, in 2017 the discovery of new TB cases totaled 145 people and an increase in 2018 of 170 people. With the discovery of this TB casse also the use of OAT is increasing because all new patients detected by TB disease both pulmonary $\mathrm{TB}$ and extrapulmonary $\mathrm{TB}$ are immediately given Category I OAT treatment. ${ }^{4}$ At present the treatment of $\mathrm{TB}$ is given in the form of packages in the form of Anti-Tuberculosis Drugs Combined Permanent Doses. TB treatment is divided into 2 phases, namely intensive phase (2-3 months) and advanced phase (4-7 months). The drug alloy used consists of a combination of primary and additional drugs. In the treatment of TB, first-line OAT is the main type of drug used. First-line drugs include isoniazid (INH), rifampicin, pyrazinamide, streptomycin, and ethambutol. The packaging of these drugs is a single tablet, presented separately, each isoniazid, rifampicin, pyrazinamide and ethambutol or it can also
Correspondence: Tiara Mayang Pratiwi Lio, D-IV Medical Laboratory Technology, STIKES Mandala Waluya Kendari, Kendari 93232, Indonesia.

Tel.: +6282292534410

E-mail: tiaramayangpratiwilio@yahoo.com,

Key words: Serum glutamic oxaloacetic transaminase, serum glutamic pyruvic transaminase, tuberculosis, Kendari.

Contributions: SR and Y, data collection and analysis; A, manuscript writing; TMPL, manuscript reviewing and references research.

Conflict of interest: The authors declare no potential conflict of interest.

Funding: Self funded.

Acknowledgements: The authors would like to thank LPPM STIKES Mandala Waluya Kendari, Study Program D-IV Medical Laboratory Technology of STIKES Mandala Waluya Kendari, and Kendari City General Hospital Kota Kendari for their help in carrying out this research.

Conference Presentation: This paper was presented at the International Conference on Infection Diseases, Biothreats, and Military Medicine (INSBIOMM), 2019 August 27-28, Surabaya.

Received for publication: 17 February 2020. Accepted for publication: 1 July 2020.

This work is licensed under a Creative Commons Attribution-NonCommercial 4.0 International License (CC BY-NC 4.0).

CCopyright: the Author(s), 2020

Licensee PAGEPress, Italy

Infectious Disease Reports 2020; 12(s1):8737 doi:10.4081/idr.2020.8737

be a fixed dose combination drug (FDC). FDC consists of 3 or 4 drugs in one tablet. OAT alloys are provided in packages, with the aim of facilitating drug administration and ensuring continuity of treatment until completion. It is one package for one patient in one treatment period. ${ }^{5}$ The advantage of giving OAT-FDC is that it makes it easier to give drugs and ensure continuity of treatment until completion, but there are some risks of side effects in patients such as impaired liver function. ${ }^{3}$

Morbidity and mortality due to TB is a serious problem mainly due to side effects caused by Anti Tuberculosis Medication (OAT). The most serious side effect of OAT is hepatotoxic. Recommended TB treatment regimens are Isoniazid $(\mathrm{H})$, Rifampicin $(\mathrm{R})$, 
Pyrazinamide (P), Ethambutol (E) and Streptomycin (S). The first 3 drugs (isoniazid, rifampicin, pyrazinamide) are hepatotoxic. OAT hepatoxicity can usually occur within the first two months of treatment The mechanism of hepatoxicity is divided into two things, intrisic (predictable) and idiosyncratic (unpredictable) reactions. Hepatotoxicity of OAT generally occurs through idiosyncratic reactions. Hepatotoxicity due to OAT causes extensive and permanent liver injury and can cause death if not detected in the initial stage. In addition to hepatotoxicity, OAT can also cause cirrhosis, liver cancer, and lead to death. ${ }^{6}$

Hepatotoxicity, gastrointestinal and neurological disorders are side effects that often occur in the treatment of tuberculosis. Hepatotoxicity is a potential complication that is almost always present in treatment. This is because the liver functions as a center for the metabolic disposition of pseudo drugs and foreign substances in the body. In hepatocytes, the drug is changed to be more hydrophilic, so that it can dissolve and can be excreted into the urine or bile. Liver lesions caused by anti-tuberculosis drugs are hepatocellular reactions that have a direct effect, namely the production of enzyme-drug complexes. This complex will then cause cell dysfunction, membrane dysfunction, and $\mathrm{T}$ cell cytotoxic response. ${ }^{7}$

According to the American Association for the Study of Liver Disease (AASLD), the parameter to determine the presence or absence of liver damage is an increase of more than three times the upper limit of nor- mal of Serum Glutamic Pyruvic Transaminase (SGPT) levels . Increased liver enzymes Serum Glutamic Oxaloacetic Transaminase (SGOT) is also considered an indicator of liver damage. In this research SGPT and SGOT examination are performed on patients with pulmonary TB who undergo treatment with intensive stage OAT. In the treatment of pulmonary TB in Kendari City Hospital, patients diagnosed with pulmonary TB were directly given Category I OAT, but the patient did not perform liver function checks first, to value SGPT and SGOT levels as markers of liver function. When the intensive treatment phase runs until the end of treatment, the patient is no longer monitored for the condition of his liver function, and is only monitored through sputum examination without monitoring SGOT and SGPT levels. Given the importance of monitoring liver function due to the side effects of OAT, researchers feel the need to do liver function tests to determine levels of SGPT and SGOT before and after the use of intensive stage of OAT.

Based on observations in patients with pulmonary TB who seek treatment at Kendari City Hospital there are a number of complaints from patients at the intensive stage OAT. These complaints resemble disorders of liver function such as shortness of breath, dizziness and nausea after consuming OAT. According to the doctor's diagnosis, the patient is suspected of having liver function disorders, but research has never been done to detect the state of liver function in patients with pulmonary $\mathrm{TB}$ in Kendari City Hospital.

\section{Materials and Methods}

\section{Types of research}

The method of this research was descriptive analysis with cross sectional approach. The sample is all patients had previously been diagnosed with TB by a doctor based on medical records by in RSUD Kota Kendari after 1-2 months OAT treatment without any sign of liver injury before were 15 people. ${ }^{4}$ Examination of SGOT and SGPT levels was performed on all samples in the Laboratory of RSUD Kota Kendari using clinical chemistry analyzer. This research was conducted on April 24 to May 18, 2019 at the Kendari City Hospital and at the Kendari City Hospital Laboratory for sample examination.

\section{Sample Criteria}

1. Inclusion Criteria

a) Patients with TB who consume intensive stage of OAT regularly since the start of treatment that is controlled through Drug Taking Supervision (PMO)

b) TB patients who have been examined for SGOT and SGPT levels before consuming OAT

2. Exclusion Criteria

a) Patients with cirrhosis of the liver

b) TB sufferers who consume alcohol

c) Not willing to be the subject of research

In this study, researchers examined blood samples to determine the state of liver

Table 1. The Result of SGOT and SGPT Levels in TB Patients who are undergoing OAT treatment in Kendari City General Hospital Kota Kendari

\begin{tabular}{|c|c|c|c|c|c|}
\hline No. & Sample & Age (year) & Gender ( $\mathrm{P}=$ female/L=male) & SGOT Level & SGPT Level \\
\hline 1 & $\mathrm{Al}$ & 27 & $P$ & 25.0 & 26.4 \\
\hline 2 & A 2 & 54 & $\mathrm{~L}$ & 13.0 & 16.3 \\
\hline 3 & A 3 & 30 & $\mathrm{~L}$ & 24.6 & 27.3 \\
\hline 4 & A 4 & 70 & $\mathrm{~L}$ & 47.5 & 50.0 \\
\hline 5 & A 5 & 45 & $P$ & 14.4 & 16.7 \\
\hline 6 & A6 & 56 & $P$ & 45.3 & 43.0 \\
\hline 7 & A7 & 50 & $\mathrm{~L}$ & 59.2 & 60.3 \\
\hline 8 & A8 & 50 & L & 14.1 & 15.7 \\
\hline 9 & A9 & 39 & $P$ & 13.0 & 15.7 \\
\hline 10 & $\mathrm{~A} 10$ & 30 & L & 13.2 & 14.7 \\
\hline 11 & All & 24 & L & 21.1 & 22.7 \\
\hline 12 & $\mathrm{~A} 12$ & 31 & P & 11.1 & 13.2 \\
\hline 13 & $\mathrm{Al} 3$ & 28 & $P$ & 15.0 & 16.4 \\
\hline 14 & Al4 & 23 & L & 14.1 & 15.3 \\
\hline 15 & $\mathrm{~A} 15$ & 46 & $\mathrm{~L}$ & 11.2 & 13.4 \\
\hline
\end{tabular}

Note: Reference value from clinical pathology laboratory of Kendari City Hospital.

a. SGPT $\leq 37 \mathrm{U} / \mathrm{L}$ (male: $\mathrm{L}) . \leq 31 \mathrm{U} / \mathrm{L}($ female: $\mathrm{P})$

b. SGOT $\leq 37 \mathrm{U} / \mathrm{L}$ (male) 
function in patients with pulmonary $\mathrm{TB}$ who consume intensive stage OAT in Kendari City Hospital. In 2019 in the period from February to March period we found 15 TB patients at 1-2 month OAT treatment. In the intensive stage patients who are diagnosed with TB are advised to consume OAT every day for 2 months. Researchers performed phlebotomy (venous blood sampling) to respondents who had met the inclusion criteria at the Kendari City Hospital and then examined the SGPT and SGOT in the laboratory of the Kendari City Hospital using clinical chemistry analyzer (Table 1).

In the study out of the 15 respondents who suffered from TB, the number of male respondents was $9(60 \%)$ while female respondents amounted to 6 people $(40 \%)$. Male espondents had the highest percentage of SGOT and SGPT enzyme levels. This is in line with research conducted by Clarasanti (2016), where from 186 data collected male respondents resulted having the highest percentage of SGPT and SGOT levels, which is around $60 \%$. This is because biotransformation in women is slower than men and acetylators in women are slower. The acetylator reaction is a reaction on the drug's metabolic pathway. The higher incidence of side effects of OAT in men is also caused by androgens, which can increase the work of liver microsomal enzymes,so that the metabolism of drugs that take place in the liver is faster.

The results of examination of SGOT and SGPT levels in 15 samples of pulmonary TB patients undergoing OAT treatment in Kendari City Hospital, 12 samples $(80 \%)$ resulted having normal SGOT and SGPT levels, while 3 samples (20\%) have abnormal SGOT levels. Patients with pulmonary TB with abnormal SGOT and SGPT were respectively 50 years old, 56 years old, and 70 years old. This shows that the percentage increase in SGOT and SGPT levels is relatively large in TB patients aged $50+$ years. This is in line with research
Khadak (2007) which states that the older a person is, the higher the risk of hepatoxicity will be, because liver function will decrease with increasing age. In the Toastman study (2010) states that hepatoxicity in pulmonary TB patients is affected by an increase in age due to decreased clearance of drugs metabolized by the CYP450 enzyme and also changes in blood flow in the liver and changes in liver size with increasing age. When those whose liver function has decreased because of old age must metabolize OAT for a long period of time, they will be more susceptible to suffering from the hepatotoxicity of OAT.

Metabolism of drugs in the liver is mainly related to drugs given orally. To get through the intestinal cell membrane, the drug must be fat-soluble, then carried to the liver, in the liver converted to dissolve in water (more polar), then excreted in urine (if the molecule is small, i.e. less than 200 mol.wt) or through bile (if the molecular weight is more than 200 mol.wt). Liver response to drug administration depends on two factors: environmental factors, in the form of high environmental temperatures, that can cause peripheral blood vessels to widen so that it can increase the working capacity of vasodilators, and genetic factors that determine the body's metabolic system and one's resistance to drugs. ${ }^{8}$

\section{Conclusions}

Anti Tuberculosis Drugs (Obat Anti Tuberkulosis/OAT), contain isoniazid, rifampicin, and pyrazinamide, and can cause severe damage to liver function. The damage caused will increase blood transaminase levels namely SGOT and SGPT which are markers for detecting liver damage. In this study, elevated levels of SGPT and SGOT due to Anti Tuberculosis (OAT) drugs did not occur in all patients and interested only 3 people, but qualitatively the effect of administering OAT significantly increased levels of SGPT and SGPT. Thus, monitoring of liver physiology remains recommended, especially for elderly patients.

\section{References}

1. Widoyono. 2011. Tropical Disease. Epidemiology, Transmission, Prevention and Eradication. Erlangga Publisher. Jakarta.

2. Tjandra Yoga Aditama. 2002. Tuberkulosis. Jakarta : IDI Publisher Foundation

3. RI Ministry of Health. 2017. National Guidelines for the Prevention of Tuberculosis 2017.Accessed on http://www.tbindonesia.or.id/opendir/B uku/bpn_p-tb_2017.pdf.

4. Health Officer of Kendari City. 2019. List of Tuberculosis Patients who are Undergoing OAT Treatment in Kendari City General Hospital Kota Kendari

5. World Health Organization. 2002. Operational Guide for National Tuberculosis Control Program on the introduction and use of fixed dose combination drugs. Geneva: World Health Organization

6. Schaberg T, Rebhan K, Lode H. 1996. Risk factors for side-effects of isoniazid, rifampin and pyrazinamide in patients hospitalized for pulmonary tuberculosis. Eur Respir J. 9:20262030.

7. Pawlowski A, Jansson M, Sköld M, Rottenberg ME, Källenius G. Tuberculosis and HIV co-infection. Plos Pathogene. 2012;8:e1002464.

8. Seng Kok-Yong and Lee Lawrence. 2015. Drug Distribution and Drug Elimination, Basic Pharmacokinetic Concepts and Some Clinical Applications, Tarek A Ahmed, IntechOpen, DOI: 10.5772/59929. 